\title{
Evaluación económica del proyecto de una pequeña industria de vigas laminadas encoladas en Brasil
}

Ernesto Augusto Garbe ernestoaugustogarbe@gmail.com Universidade Federal do Paraná (UFPR), Curitiba, Paraná, Brasil

Jorge Luis Monteiro de Matos jmatos.ufpr@gmail.com Universidade Federal do Paraná (UFPR), Curitiba, Paraná, Brasil

Delcio Pereira

delcio.pereira@udesc.br

Universidade do Estado de Santa Catarina (UDESC), São Bento do Sul, Santa Catarina, Brasil

\section{José Guilherme Prata}

jgprata@gmail.com

Universidade Federal do Paraná (UFPR), Curitiba, Paraná, Brasi

\begin{abstract}
La evaluación económica de un proyecto de industria es fundamental para disminuir la probabilidad de fracaso de una inversión. Evaluar el resultado potencial de una pequeña fábrica de madera laminada encolada en Brasil fue el principal objetivo del presente trabajo, siendo necesario para eso elaborar un flujo de caja previsto con base en valores de facturación y salidas de caja para costeo de materias primas y de la operación. Se identificaron índices de evaluación económica, siendo el principal de ellos la Tasa Interna de Retorno de la inversión, de un 45\%, revelando un Valor Presente Líquido positivo y un Payback de poco más de 2 años. Estos indicadores hacen el proyecto viable y bastante atractivo a los ojos de los inversionistas. También se evaluó el riesgo de la inversión, con base en un análisis de sensibilidad a los factores de facturación y costo de la materia prima, siendo evidenciado un bajo riesgo.
\end{abstract}

PALAVRAS-CHAVE: Madera laminada. Glulam. Viabilidad económica. 


\section{INTRODUCCIÓN}

Una inversión puede ser designada como una propuesta de aplicación de recursos escasos que poseen aplicaciones alternativas a un negocio, como también un sacrificio hecho en el momento para obtener un beneficio futuro (REMER; NIETO, 1995). De acuerdo con Queiroz (2001), el análisis de inversión es fundamental en la asignación eficaz de recursos escasos en el ambiente organizacional.

Cuando los gestores de una organización piensan en invertir en equipos, ampliar el establecimiento o, incluso reducir los costos (WERNKE, 2000), necesitan efectuar un análisis de inversión, para no realizar acciones equivocadas que puedan perjudicar el futuro de la empresa. Ante estos escenarios, este trabajo buscó respuesta al siguiente cuestionamiento: ¿Cuáles son los resultados de la inversión en una pequeña industria del sector de Vigas Laminadas Encoladas, instalada en la región de Curitiba PR-Brasil.

El objetivo de este trabajo es dar soporte a la toma de decisión de una inversión en una pequeña unidad de producción de vigas laminadas encoladas, identificando la viabilidad económica de este capital invertido. Los objetivos específicos son:

- Determinación de los índices para el análisis de la inversión y la evaluación de los resultados previstos en un horizonte de 5 años.

- Identificación del riesgo de invertir en una unidad de producción de vigas laminadas en Brasil y el impacto que éste representa en una inversión.

El análisis de inversiones será la base que subsidiará la toma de decisiones sobre montar un negocio o no. Este análisis considera indices basados en un flujo de caja previsto en un horizonte de planificación, así como la incertidumbre que existe, lo que genera un riesgo.

Análisis de inversiones, según FLEISCHER (1973) es el proceso de selección entre propuestas alternativas de inversión, a fin de maximizar los beneficios a largo plazo.

La tasa de descuento que se utilizará puede ser una tasa mínima de atractivo, que puede ser definida por los inversores o los analistas. Su concepto es de ser la mejor alternativa de inversión con el menor grado de riesgo disponible para la aplicación (SOUZA; CLEMENTE, 2004). Esta tasa según Damodaran (2010), se compone de tres componentes básicos:

a) costo de oportunidad: remuneración obtenida en alternativas que no se analizan. Ejemplo: cuaderno de ahorro, fondo de inversión;

b) riesgo del negocio: la ganancia tiene que remunerar el riesgo inherente a una nueva acción. Cuanto mayor sea el riesgo, mayor será la remuneración esperada; $y$,

c) liquidez: capacidad o velocidad en que se puede salir de una posición en el mercado para asumir otra.

Para identificar si el proyecto de inversión va a atender a las metas establecidas por los administradores y accionistas, es fundamental conocer los índices determinados por la Tasa Interna de Retorno (TIR) en la viabilidad del resultado financiero presentada en forma de tasa. La interpretación de esta es 
por medio de comparación con la TMA, si el resultado de la TIR es mayor o igual a la TMA, significa que la inversión es viable, dentro de los parámetros establecidos por los gestores.

El valor presente neto - VPN es el resultado que la inversión proporcionará al final del proyecto, utilizando la TMA. Sin embargo, representa el valor de la caja proyectada, disminuyendo el costo, donde se identifica el resultado financiero de la inversión. En el caso de Casarotto y Kopittke (2008, p. 116), VPN se compone de un cálculo simple donde, "en lugar de distribuir la inversión inicial durante su vida (costo de recuperación del capital), se deben sumar los demás valores del flujo de caja para sumarlos a la inversión inicial de cada alternativa. Se elige aquella que presente mejor Valor Presente Neto".

Para examinar un proyecto, uno de los factores es identificar el tiempo necesario para alcanzar el retorno sobre la inversión. Para ello es necesario aplicar el método payback, encontrando así el tiempo que se deben considerar los flujos de caja líquido generados, sustraídos de la inversión inicial, para evaluar su plazo de retorno.

De acuerdo a Ross (2000, p218), el "payback es el período requerido para que la inversión genere flujos de efectivo suficientes para recuperar el costo inicial". El payback no debe ser una única fuente de análisis, como mencionan Motta y Calôba (2002, p97) "debe considerarse con reservas, apenas como un indicador, no sirviendo de selección entre alternativas de inversión". Su principal ventaja es tener en cuenta el tiempo en que se recuperará el dinero del inversionista, evaluando su necesidad financiera, para que se mantenga la inversión hasta el momento de generar las cajas futuras positivas. De acuerdo con Casarotto y Kopittke (2008, p.125), "el principal método no exacto mide el tiempo necesario para que la suma de las cuotas anuales sea igual a la inversión inicial". Generalmente se puede decir que registra el tiempo promedio para que los flujos de caja se equiparen al valor de la inversión. El payback tradicional presupone una desventaja, ya que proyectar los flujos de efectivo en el tiempo no lleva a la corrección de los valores monetarios. Al considerar el payback descontado, se observa el real valor del dinero en el tiempo, obteniendo una estimación financiera más realista.

Los problemas de aplicación de capital, según FLEISCHER (1973), invariablemente se relacionan con el futuro. El pasado es de interés sólo en la medida en que proporciona información sobre las cantidades y el tiempo esperado de los futuros flujos de caja. En vista de este énfasis, es necesario, dirigirse al problema de la incertidumbre que está siempre asociada a las estimaciones de los futuros eventos. Una de las técnicas que se pueden utilizar para considerar la incertidumbre es el análisis de sensibilidad FLEISCHER (1973).

Cuando un pequeño cambio en el valor de una estimación particular resulta en cambio en la elección de la alternativa, se dice que la decisión es sensible a esa estimación. Por el contrario, la decisión es insensible a la estimación cuando las variaciones relativamente amplias en la estimación original no modifican la solución original FLEISCHER (1973).

Según HARRISON (1978), este es un enfoque y no una técnica bien definida. Este autor explica así el enfoque del análisis de sensibilidad: para cada una de las variables asociadas a un proyecto, se especifica no sólo su valor más probable, sino también sus límites superior e inferior de variación. A continuación, se 
calculan los flujos de caja obtenidos, si estos valores límite realmente ocurren. Con ello se pasa a tener tres conjuntos de flujos de caja previstos para el proyecto: uno correspondiente a la estimación en la media, y dos más, correspondientes a los límites inferior y superior. Se calcula entonces, los valores del Valor Presente Neto (o Tasa Interna de Retorno, lo que sea más apropiado) para cada uno de los tres conjuntos de valores. Se tiene, así, un conjunto de valores posibles para el Valor Presente Neto en función del comportamiento de una variable HARRISON (1978).

La elaboración del flujo se realiza a partir de la Demostración de Resultado del Ejercicio, restando las salidas de las entradas. Con el conjunto de los valores de los flujos encontrados período a período, es posible calcular diversos indicadores del proyecto. Motta (2011) sugiere un modelo de flujo de caja que contiene las entradas y salidas en el horizonte de cinco años, así como al final algunos indicadores de evaluación de la viabilidad económica del proyecto analizado.

De hecho, la importancia de los flujos de caja en las decisiones de inversión es ampliamente reconocida. NETO (2012) evidencia que el aspecto más importante de una decisión de inversión se centra en el dimensionamiento de los flujos previstos de caja a ser producidos por las propuestas en análisis. En realidad, la confiabilidad sobre los resultados de una determinada inversión depende en gran medida del ajuste con el que se han proyectado sus flujos de entradas y salidas de caja.

Se debe reforzar la regla básica de que todo proyecto de inversión se evalúa en términos de flujo de caja, en lugar de basarse en los beneficios. Prette (2014) afirma que esa elección se justifica ya que es por medio de los resultados de caja que la empresa asume efectiva capacidad de pago y reaplicación de los beneficios generados en la decisión de inversiones. En otras palabras, es mediante los flujos de caja, y no los beneficios, que se mide el potencial efectivo de la empresa de implementar sus decisiones financieras fundamentales (inversión, financiamiento y distribución de dividendos). De esta forma, son los flujos de caja, y no otra medida contable cualquiera sea el resultado, que se constituyen en la información más relevante para el proceso de análisis de inversiones.

\section{MATERIALES Y MÉTODOS}

La metodología utilizada para el presente trabajo sirvió para determinar la capacidad de producción, facturación y costos, así como las inversiones necesarias para el proceso y equipamientos, instalaciones y capital de trabajo. Estos valores fueron utilizados en la elaboración de flujo de caja, del cual se extrajeron los indicadores económicos.

Considerando que el presente trabajo tiene como objetivo la evaluación económica de un proyecto de producción de pequeña escala de vigas laminada encoladas - VLE, se han estudiado los principales métodos existentes para ello.

Este estudio identificó algunos índices basados en un flujo de caja para análisis, así como las fórmulas para definir los índices y las reglas para verificar la viabilidad económica basada en los índices. 


\section{ELABORACIÓN DEL FLUJO DE CAJA PREVISTO PARA UNA INDUSTRIA DE VLE}

Para la elaboración del flujo de caja se consideraron las entradas y salidas de caja (dinero) previstas para los cinco primeros años de funcionamiento del emprendimiento. Se consideró como modelo para el desarrollo de la propuesta de flujo de caja ideado por Motta (2011).

\section{ENTRADAS DE CAJA}

Para las entradas de caja, se consideró la utilización de capital propio, no colocando valores referentes a préstamos. En las entradas, se consideraron los valores relativos a las ventas de productos, siendo para ello necesario el dimensionamiento de la industria; la definición de los productos y los precios; y el dimensionamiento de la producción y la capacidad utilizada prevista. Estos elementos se explican a continuación:

\section{Dimensionamiento de la Industria}

La capacidad de producción debe satisfacer la demanda prevista de VLE determinada en un estudio de mercado. El estudio fue basado en datos proporcionados por revisiones de literatura y validados en entrevistas a empresarios productores de vigas laminadas. Este estudio evaluó el potencial de ventas existente en Brasil, así como la cantidad producida por la competencia, la diferencia entre los valores basó el dimensionamiento de la industria, utilizando una saturación de mercado inferior al $15 \%$. Estos datos se consideraron en la composición del flujo de caja.

\section{Definición de los Productos y Precios}

En el mismo estudio de mercado se determinaron los productos de VLE para la comercialización, así como el precio de la competencia. Estos fueron los valores considerados en la facturación de la empresa en ventas de productos y que constituyen la principal fuente de entrada en el flujo de caja estudiado.

\section{Dimensionamiento de la Producción y Capacidad Utilizada Prevista}

La producción en los años iniciales de la industria fue considerada con capacidad inferior al $100 \%$, o sea, no estando en su plena capacidad de productividad. Esto puede ocurrir por diversos motivos entre ellos, la falta de ventas, pérdidas de productividad por ajustes de equipos y entrenamiento de obreros, entre otros. Para el primer año de producción, se consideró la capacidad del $65 \%$, para el segundo año $80 \%$ y del tercer año en adelante el $100 \%$, según lo propuesto por Delespinasse (1994). En el año cero se consideró la producción nula. 


\section{SALIDAS DE CAJA}

Las salidas que se incluyeron en el flujo de caja previsto se refieren a las inversiones iniciales, así como los costes fijos y variables de producción y ventas de las vigas laminadas.

\section{Inversión}

Las inversiones necesarias para el proyecto de la fábrica de VLE se determinaron basándose en la entrevista con el empresario de la rama y los datos de revisión de bibliografías, donde se enumeraron los equipos, la necesidad de capital de trabajo y otros necesarios para el funcionamiento. Estos elementos fueron sumados y colocados como la primera salida de caja en el año cero, simulando la compra de los materiales.

\section{Materias Primas y Insumos}

Las piezas de vigas laminadas se producen con laninas de madera con superficies lisas y pegadas superficialmente formando un bloque de dimensiones más grandes. La cola utilizada debe tener resistencia estructural.

Se consideró la capacidad prevista anualmente para producción de VLE, así como las pérdidas del proceso, se enumeraron las materias primas e insumos necesarios (cola y madera) para esta producción. Estas cantidades se transformaron en valores de compra basados en los precios identificados en el estudio de mercado propuesto por Garbe (2017).

\section{Mano de Obra}

Para cada parte del proceso productivo y sus equipos utilizados, son necesarios obreros para desempeñar sus funciones. En base a esto se determinaron según la entrevista con el empresario de la rama, determinando el número de obreros necesarios y los salarios con cargos. Estos valores se sumaron y se determinó el total de la nómina con cargo anual. Este valor se consideró como salida de caja en todos los años de producción del horizonte de 5 años del flujo de caja.

\section{Administrativos}

En base a la entrevista con empresario de la industria de vigas laminadas se obtuvo el total de costos administrativos relativos al período de un mes. Estos valores se transformaron en anuales. Los costos administrativos son aquellos que no impactan directamente en el producto pero necesitan existir para que la fábrica esté en pleno funcionamiento. Los principales costos identificados son agua, alquiler, contador, teléfono, entre otros. En la entrevista fueron identificados todos aquellos que inciden en la empresa, según el empresario.

Según Delespinasse (1994), estos gastos están representados por los siguientes elementos: alquileres; alimentación del trabajador; mantenimiento y 
conservación de muebles y utensilios; material de limpieza y conservación; seguros; gastos de vehículos pequeños; sueldo del dueño; viajes y estadías; teléfonos; internet; legalización, libros y documentos; periódicos y revistas; contribución sindical; donaciones y contribuciones; honorarios profesionales; material de oficina y publicidade.

\section{Impuestos y Costos de Ventas}

Para la tributación incidente en el emprendimiento estudiado se realizo una entrevista con el contador responsable de la liquidación de los impuestos generados. Según la entrevista se identificó el sistema tributario, así como el porcentaje de impuestos incidentes sobre la facturación.

Para los costos de ventas los valores levantados se basaron en una entrevista con un empresario productor de vigas laminadas. En la entrevista se levantaron todos los costos de ventas y se hizo una proporción de éste en función de la facturación. Los valores representaron un porcentaje de la facturación y se añadieron a los impuestos para ser insertados en el flujo de caja.

\section{Depreciación}

Considerado como el hecho físico de perecimiento del material (equipamiento y edificaciones), generando un hecho económico y la consecuente necesidad de formación de reserva económica y financiera para la sustitución del equipo, al término de su vida útil, se utilizan como tasas de depreciación las permitidas por los ingresos federales de Brasil, es decir, del $10 \%$ anual para equipos y máquinas y del $4 \%$ para edificaciones.

\section{DETERMINACIÓN DE LOS INDICES DE EVALUACIÓN ECONÓMICA}

Los principales índices de evaluación económica de una determinada inversión son: valor presente neto - VPN; Tasa interna de retorno - TIR; TIR MODIFICADA; Plazo de retorno de la inversión - Payback; Payback descontado. Para el análisis completo se deben evaluar los índices basados en una tasa mínima de atractivo - TMA. Estos valores se identificaron con el flujo de caja y la TMA en base a entrevista con un empresario.

Para obtener un atractivo económico en el proyecto se deben obtener los índices y los mismos deben ser evaluados separadamente. Habrá viabilidad económica cuando el valor presente neto - VPN sea positivo; Tasa interna de retorno - TIR y; TIR MODIFICADA sean superiores a la tasa mínima de atractivo; los indicadores de plazo de retorno de la inversión - Payback; y el Payback descontado son complementarios al análisis.

\section{EVALUACIÓN DE LA VIABILIDAD ECONÓMICA}

La viabilidad económica de este proyecto fue evaluada comparando los indices obtenidos para el flujo de caja previsto, con los valores de referencia 
como se explicó anteriormente. Los principales índices que determinan la viabilidad son VPN; TIR y TIR MODIFICADA.

\section{IDENTIFICACIÓN DE LOS FACTORES DE RIESGO EN LA PRESENTE INVERSIÓN}

Los factores de riesgo son aquellos que pueden afectar negativamente el resultado del negocio en términos de caja. En este ítem se levantaron los factores que más pueden impactar en el flujo de caja y se hizo una simulación de análisis de riesgo, variando para más y para menos cada uno de los ítems y creándose un cuadro denominado análisis de sensibilidad del proyecto. En este cuadro, en las columnas se coloca uno de los factores y en las líneas el otro factor de riesgo. En la tabla se simula el resultado de la inversión con las variaciones presentadas. Se muestra el indicador TIR MODIFICADA, la cual, siendo menor que la TMA indica situación de amenaza al proyecto de acuerdo con la situación simulada.

\section{DETERMINAR EL IMPACTO DEL RIESGO EN EL RESULTADO DEL NEGOCIO}

Con base en lo que se obtuvo en la tabla de análisis de sensibilidad se hace un análisis cualitativo del ejemplificado y verificado riesgo del negocio. Indices de TIR MODIFICADA cercanos o inferiores a TMA indican mayor riesgo al negocio.

\section{RESULTADOS Y DISCUSIÓN}

En este ítem se siguen los passos determinados en la metodología y se evalúa al final la viabilidad económica del proyecto, así como la sensibilidad de éste a los riesgos existentes.

\section{ELABORACIÓN DEL FLUJO DE CAJA PREVISTO PARA UNA INDUSTRIA DE VLE}

En un horizonte de 5 años es posible evaluar el resultado de la inversión en una pequeña fábrica de vigas laminadas. Se consideraron datos de la entrevista con el empresario de la rama y los datos de revisión de bibliografías, los cuales se asignaron anualmente. El flujo de caja consideró las entradas y salidas como se muestra a continuación.

\section{ENTRADAS DE CAJA}

Las entradas de caja son los valores previstos en el flujo de caja referentes principalmente a la facturación. Para ello se dimensionó el tamaño de la industria, productos y precios de comercialización y la capacidad utilizada. La multiplicación simple de la cantidad producida por la capacidad utilizada y por el precio unitario nos indica la previsión de facturación. Este valor se encuentra en la tabla de flujo de caja en el campo entradas - ventas.

Se consideró una fábrica con capacidad de producción de $1.260 \mathrm{~m}^{3}$ anuales, según se cree puede absorber el mercado de madera laminada. Este valor representa menos del $10 \%$ del potencial de mercado en un análisis conservador, o sea la probabilidad de su comercialización es bastante alta. Esto representa una 
producción de $105 \mathrm{~m}^{3}$ mensuales de VLE. Esta capacidad de producción será el máximo producido cuando esté en su capacidad del $100 \%$.

El producto principal considerado es las vigas laminadas encoladas, con longitudes entre 6 a 20 metros y de formato rectilíneo, es decir, sin curvatura. Como se ha estudiado, se ha identificado para este tipo de producto un precio de $\mathrm{R} \$ 4.000$ por metro cúbico de MLC. Este fue el valor considerado para la venta y por lo tanto considerado como entradas en el flujo de caja. Para fines de comparación, el valor de la conversión de cambio a dolar estadunidense es de $\mathrm{R} \$ 3,13$, en agosto de 2017.

Se consideraron los porcentajes de $0 \%$ de producción en el año cero (implantación), el $65 \%$ en el primer año de operaciones, y el $80 \%$ en el segundo año, alcanzando el $100 \%$ sólo del tercer año en adelante, según lo propuesto por Delespinasse (1995). Esta reducción disminuye la facturación de la empresa y revela una producción de aproximadamente $68 \mathrm{~m}^{3}$ mensuales en el primer año, $84 \mathrm{~m}^{3}$ mensuales en el segundo año y $105 \mathrm{~m}^{3}$ en el tercer año en adelante.

El valor principal considerado como entradas fue referente a las ventas del período anual o facturación, que está representado por el valor de venta de $\mathrm{R} \$ 4.000$ por metro cúbico, que se obtiene de la producción anual de $1.260 \mathrm{~m}^{3}$. En los dos primeros años de producción, se consideraron valores de producción por debajo de la capacidad máxima, lo que simula la realidad en las empresas. Se consideraron respectivamente a los AÑO 1 y AÑO 2, 35\% por debajo y 20\% por debajo, siendo del AÑO 3 en adelante considerada la capacidad del 100\% de producción y ventas. No se consideraron ventas en el AÑO 0.

\section{SALIDAS DE CAJA}

Para las salidas de caja se consideraron separadamente diversos elementos del flujo de caja, lo que facilita el entendimiento y la evaluación de qué factores impactan con mayores valores en el cuadro anual. Se contabilizaron las inversiones; materias primas e insumos; mano de obra; costos administrativos; impuestos y costos de ventas; y la depreciación.

En cuanto a las salidas referentes a la operación, no se introdujeron valores en el AÑO 0 debido al hecho de que las compras de materias primas, mano de obra y otros costos administrativos ya se consideraron en la inversión inicial, constando como necesidad de capital de trabajo y preoperativos.

Para la inversión inicial fueron, según entrevista y datos propuestos por Garbe (2017), considerados valores de R\$610 por metro cúbico de producción anual y una producción anual de 1.260 metros cúbicos. Esto totaliza una inversión inicial de R\$763.065. En el flujo de caja este valor fue asignado en las salidas, para facilitar la visualización en el año cero. Siendo los equipos necesarios ( $R \$ 504.279)$; los costos preoperativos ( $R \$ 158.000)$ y el capital de giro ( $R \$ 100.786$ ), se totaliza una inversión total del orden de $R \$ 763.065$, sin considerar la construcción, ya que se utilizará un galpón alquilado.

Las salidas de caja referentes a las compras de materias primas e insumos fueron asignadas anualmente y también consideraron los valores de producción por debajo de la capacidad máxima, similar a lo ocurrido en las ventas. Para valores de costos se consideraron para la madera de Pinus encolada con 
melamina urea formaldehído - MUF el valor de $\mathrm{R} \$ 712,06$ por metro cúbico. Se agregó el factor de aprovechamiento del 75\%, considerando que, según datos de empresas de VLE, el $25 \%$ de las materias primas e insumos en promedio se pierden en el procesamiento, datos propuestos por Garbe (2017) validados por entrevista a empresario de la rama.

Para la mano de obra, según entrevista, fueron considerados 16 obreros, los cuales efectuaron una hoja de pagos con cargos laborales en el orden de $\mathrm{R} \$ 38.000$ mensuales, lo que totalizan $\mathrm{R} \$ 456.000$ anuales.

En el caso de gastos administrativos, se consideraron, según el empresario de VLE y los datos obtenidos en encuestas, valores aproximados de cuentas mensuales de agua ( $R \$ 100)$; electricidad ( $R \$ 1.000)$; teléfono e internet ( $R \$ 300)$; contador (R\$1.000); ( $\mathrm{R} \$ 7.500)$; combustible ( $\mathrm{R} \$ 1.000)$; mantenimiento predial, vehículos y equipos ( $R \$ 500)$. Estos valores totalizan mensualmente el valor de $R \$ 11.400$, lo que representa anualmente la suma de $R \$ 136.800$.

Para finalizar el flujo de caja previsto se consideraron los impuestos y gastos con la comercialización de los productos. Estos valores se obtienen, según la empresa productora de VLC junto con su empresa de contabilidad, directamente sobre el valor facturado, pudiéndose considerar para impuestos el Simples (Impuesto de Brasil para las Pequeñas Empresas), considerado en este caso aproximadamente en un $16 \%$, para comisión de representante $10 \%$, para comisiones extras para arquitectos e ingenieros proyectistas $10 \%$, fletes $5 \%$, para incumplimiento $2 \%$ y devoluciones $2 \%$.

La depreciación del presente proyecto afectará principalmente a los equipos, ya que el lugar será alquilado. Por lo tanto se considera sólo el $10 \%$ de depreciación anual sobre el valor de $\mathrm{R} \$ 504.279$ invertidos en equipos. En total se pueden considerar anualmente $\mathrm{R} \$ 50.428$ de depreciación de los equipos.

\section{FLUJO DE CAJA PREVISTO PARA LA INDUSTRIA DE VLE}

Al unir todos estos valores de flujos de entradas y salidas, compilados en un horizonte de 5 años, podemos obtener en la Tabla 1 el flujo de caja previsto para 5 años de operación de una pequeña unidad productora de vigas laminadas, instalada en los alrededores de la región de Curitiba-PR.

Tabela 1 - Flujo de caja en reales (R\$) de una industria productora de VLE

\begin{tabular}{ccccc} 
AÑ̃S & AÑ̃O O & AÑ̃ 1 & AÑO 2 & AÑO $3^{*}$ \\
ENTRADAS TOTAL & - & $\mathbf{3 . 2 7 6 . 0 0 0}$ & $\mathbf{4 . 0 3 2 . 0 0 0}$ & $\mathbf{5 . 0 4 0 . 0 0 0}$ \\
Facturación Ventas & - & 3.276 .000 & 4.032 .000 & 5.040 .000 \\
& & & & \\
SALIDAS TOTAL & $\mathbf{7 6 3 . 0 6 5}$ & $\mathbf{2 . 8 9 4 . 9 9 8}$ & $\mathbf{3 . 4 1 4 . 6 3 7}$ & $\mathbf{4 . 1 0 7 . 4 8 9}$ \\
Inversión Inicial & 763065 & 0 & 0 & 0 \\
Mat. Prima Insumos & - & 777.569 & 957.008 & 1.196 .261 \\
Mano de Obra & - & 456.000 & 456.000 & 456.000 \\
Administrativos & - & 136.800 & 136.800 & 136.800 \\
Depreciación & - & 50.428 & 50.428 & 50.428 \\
Impuestos e Ventas & - & 1.474 .200 & 1.814 .400 & 2.268 .000 \\
RESULT. ANUAL & $\mathbf{- 7 6 3 . 0 6 5}$ & $\mathbf{3 8 1 . 0 0 2}$ & $\mathbf{6 1 7 . 3 6 3}$ & $\mathbf{9 3 2 . 5 1 1}$ \\
\hline
\end{tabular}

*Los años 4 y 5 son similares a los datos del año 3.

Fonte: O Autor 
La Tabla 1 muestra el flujo de caja del horizonte de planificación de 5 años de operación y el año cero, considerado para instalación de la industria. Se puede verificar que el cuadro es negativo sólo en el año cero, siendo positivo en los demás años. Esto todavía no significa que la inversión es viable, pero es un indicador de que no habrá falta de caja en los años evaluados.

Se puede verificar también que los valores referentes a los impuestos y costos de ventas son los más representativos, seguidos por la materia prima y insumos y la mano de obra.

\section{VIABILIDAD DE LA INVERSIÓN}

Los índices siguientes se calcularon según la literatura en hojas de excel y considerando una tasa mínima de atractivo - TMA del 20\% podemos constatar que el valor presente neto - VPN es de $\mathrm{R} \$ 1.122 .725,87$, siendo este positivo, se puede decir que es un indicador de viabilidad económica. La tasa interna de retorno de la inversión TIR es igual al 74\%, constatando un reembolso de 1 año y 8 meses para devolver la inversión. Para mayor confiabilidad, se constata que la TIR MODIFICADA es del 45\%, aún mayor que la TMA del $20 \%$ considerada, por lo tanto el proyecto es viable económicamente y determina un plazo de Payback Descontado de 2 años y 1 mes. El retorno de la inversión, si se consideran inversiones en ahorro, sería de al menos 10 a 14 años para fines de comparación.

\section{FACTORES DE RIESGO DE INVERSIÓN}

Al analizar el flujo de caja se puede decir que la facturación es un factor de riesgo al negocio, pues las variaciones que ocurren en éste pueden impactar directamente en el resultado. También se obtiene que el costo de la materia prima y insumos puede impactar en los resultados, por lo que a continuación se hace un análisis de sensibilidad del negocio en función de estas dos variables.

Se han variado para más y para menos los factores de riesgo facturación, materia prima e insumos. El resultado fue reevaluado para cada simulación y se obtuvieron nuevos valores de TIR MODIFICADA, las cuales fueron colocadas en la Tabla 2.

Tabla 2 - Evaluación de la sensibilidad del proyecto medida en tir modificada a los riesgos identificados: faturación y materias primas y insumos

\begin{tabular}{cccccc} 
FATURACIÓN & $-20 \%$ & $-10 \%$ & $0 \%$ & $+10 \%$ & $+20 \%$ \\
$\begin{array}{c}\text { Costo Materia Prima y } \\
\text { Insumos }\end{array}$ & & & & & \\
$+20 \%$ & -32 & $1 \%$ & $26 \%$ & $48 \%$ & $69 \%$ \\
$+10 \%$ & $-16 \%$ & $12 \%$ & $35 \%$ & $57 \%$ & $78 \%$ \\
$0 \%$ & $-3 \%$ & $22 \%$ & $45 \%$ & $66 \%$ & $86 \%$ \\
$-10 \%$ & $9 \%$ & $32 \%$ & $54 \%$ & $75 \%$ & $95 \%$ \\
$-20 \%$ & $19 \%$ & 42 & 63 & $84 \%$ & $104 \%$ \\
\hline
\end{tabular}

Fonte: O Autor 
Se puede decir que la inversión es de riesgo moderado a bajo, pues para que haya inviabilización del proyecto por TIR MODIFICADA inferior a TMA es necesario que ambas variables sean modificadas conjuntamente en un $10 \%$ de forma negativa, o sea, si sólo unas de las variables que se analizan cambia en un 10\%, empeorando el escenario, aún así el proyecto seguirá siendo viable.

También puede decirse que la tasa de retorno evaluada es más sensible a la variación de la facturación que la variación del costo de la materia prima e insumos. Este es el factor más preocupante.

\section{CONCLUSIONES}

De acuerdo con los resultados obtenidos en este trabajo, es posible presentar las siguientes conclusiones:

- Los indices para el análisis de la inversión y la evaluación de los resultados previstos en un horizonte de 5 años hacen claro el análisis de viabilidad económica.

- El proyecto es viable económicamente y tiene retorno de la inversión de 2 años y 1 mes.

- El análisis de sensibilidad deja clara la identificación del riesgo de invertir en una unidad de producción de vigas laminadas en el sur de Brasil y el impacto que éste representa en una inversión.

- El riesgo de la inversión es más sensible en función de la variación de la facturación que en función de otros factores, pero se puede decir que la sensibilidad es baja y por lo tanto el riesgo también. 


\title{
Economic evaluation of an small industry project of glulam in Brazil
}

\begin{abstract}
The economic appraisal of an industry project is critical to lowering the chance of failure of an investment. Evaluating the potential result of a small factory of glued laminated wood in Brazil was the main objective of the present work, being necessary for this to elaborate a predicted cash flow based on values of billing and cash outflows for the costing of raw materials and operation. Economic valuation indices were identified, the main one being the Internal Rate of Return on investment, which was $45 \%$, revealing a positive Net Present Value and a Payback of just over 2 years. These indicators make the project feasible and attractive in the eyes of investors. The investment risk was also evaluated, based on an analysis of sensitivity to the factors of invoicing and cost of raw material, evidencing a low risk.
\end{abstract}

KEYWORDS: Laminated Wood Beams. Glulam. Economic Viability for Feasability. 


\section{REFERÊNCIAS}

CASAROTTO FILHO, N. C. \& KOPITTKE, B. H. 2008. Análise de investimentos. 10ā Edição. São Paulo: Atlas, Brasil.

DAMODARAN, A. 2010. Avaliação de investimentos. 2ª Edição. Rio de Janeiro: Qualitymark, Brasil.

DELESPINASSE, B. F. M. 1995. Simulação de análise de investimentos na indústria de compensados no brasil. Dissertação do Curso de Pós-Graduação em Engenharia Florestal - UFPR. Curitiba-PR, Brasil.

FLEISCHER, G.A. 1973. Teoria da aplicação do Capital. Um estudo das decisões de investimento. S. Paulo. E. Blücher, Brasil.

GARBE, E.A. 2017. Avaliação da viabilidade econômica da produção de vigas laminadas coladas de diferentes espécies e adesivos. Disponível em: www.artigos.com. Acesso em: 23/07/2018.

HARRISON, I. W. Avaliação de projetos de investimentos. São Paulo : McGrawHill, Brasil.

MOTTA, R. R.\& CALÔBA, G. M. 2002. Análise de investimentos: tomada de decisão em projetos industriais. São Paulo: Editora Atlas, Brasil.

MOTTA, R. R.; CALÔBA, G. M. 2011. Análise de investimentos: tomada de decisão em projetos industriais. 1. ed. 7. reimpr. São Paulo: Atlas, Brasil.

NETO, A. A. 2012. Finanças corporativas e valor. 6. ed. São Paulo: Atlas, Brasil.

PRETTE, B. G., CARDOSO, L. S. 2014. Análise de Viabilidade Econômica de Implantação de um Novo Estabelecimento de Comércio de Produtos Naturais. Rio de Janeiro, Brasil: UFRJ / Escola Politécnica.

REMER, D. S. \& NIETO, A. P. A 1995. Compendium and comparison of 25 project evaluation techniques. Part 1: Net present value and rate of return methods. International Journal of Production Economics, v. 42, n. 1. crossref

ROSS, S. A. 2000. Principio de administração financeira. 2a Edição.. São Paulo: Atlas, Brasil. 
SOUZA, A. \& CLEMENTE, A. 2004. Decisões financeiras e análise de

investimentos: fundamentos, técnicas e aplicações. 5a Edição.São Paulo: Atlas,

Brasil.

Recebido: 01 Ago. 2019

Aprovado: 13 Out. 2020

DOI: 10.3895/gi.v16n4.10434

Como citar:

GARBE, E.A.; MATOS J.L.M; PEREIRA D.; PRATA J.G. Evaluación económica del proyecto de una pequeña

industria de vigas laminadas encoladas en Brasil. R. Gest. Industr., Ponta Grossa, v. 16, n. 4, p. 146-160,

Out./Dez. 2020. Disponivel em: https://periodicos.utfpr.edu.br/revistagi

Correspondência:

Ernesto Augusto Garbe

Rua Augusto Wunderwald, número 1538, Bairro Centenário, São Bento do Sul, Santa Catarina, Brasil.

Direito autoral: Este artigo está licenciado sob os termos da Licença Creative Commons-Atribuição 4.0

Internacional.

(c) (1) 